\title{
Identification of ubiquinones and menaquinones in activated sludge by liquid chromatography-atmospheric pressure chemical ionization mass spectrometry
}

\author{
Meng-chun Gao ${ }^{\mathrm{a}}$, Min Yang ${ }^{\mathrm{a}, *}$, Jian-ying $\mathrm{Hu}^{\mathrm{b}}$, Bing Shao ${ }^{\mathrm{a}, \mathrm{b}}$, Hai-feng Zhang ${ }^{\mathrm{b}}$, \\ Hong-yan $\mathrm{Li}^{\mathrm{a}}$ \\ ${ }^{a}$ State Key Laboratory of Environmental Aquatic Chemistry, Research Center for Eco-Environmental Sciences, Chinese Academy of \\ Sciences, P.O. Box 2871, Beijing 100085, China \\ ${ }^{\mathrm{b}}$ College of Environmental Science, Peking University, Beijing 100871, China
}

Received 20 November 2002; received in revised form 8 May 2003; accepted 21 May 2003

\begin{abstract}
A sensitive analytical method has been developed for identification of ubiquinones (UQ- $n\left(\mathrm{H}_{x}\right)$ ) and menaquinones $\left(\mathrm{MK}-n\left(\mathrm{H}_{x}\right)\right)$ in activated sludge by liquid chromatography-atmospheric pressure chemical ionization mass spectrometry in negative mode (LC-NI-APCI-MS). Extraction and clean-up of samples were carried out on Sep-Pak Plus Silica solid-phase extraction cartridges. Complete separation of quinones was achieved with an ODS analytical column and using isopropyl ether-methanol $(17: 83, \mathrm{v} / \mathrm{v})$ as the mobile phase. The compositions of ubiquinones and menaquinones were determined directly using combined information on retention time, the molecular ion mass and fragment ion masses. The lowest instrument quantitative detection limits $\left(\mathrm{LOD}_{\text {inst }}\right)$ for UQ-6, UQ-10, and Vitamin $\mathrm{K} 1$ were estimated to be $0.4,4$ and $0.12 \mathrm{ng}$ $(S / N=10)$ using LC-NI-APCI-MS in SIM mode, and the lowest method detection limits $\left(\mathrm{LOD}_{\text {meth }}\right)$ achieved by spiking experiment were estimated to be $0.2,2$ and $0.06 \mu \mathrm{g} / \mathrm{g}$ for UQ-6, UQ-10 and Vitamin K1, respectively. On the other hand, the LOD $_{\text {inst }}$ for UQ-6, UQ-10, and Vitamin K1 were estimated to be 10, 100 and $2 \mathrm{ng}(S / N=10)$ using LC-NI-APCI-MS in full-scan mode, and the $\mathrm{LOD}_{\text {meth }}$ were estimated to be 7, 60 and $1.2 \mu \mathrm{g} / \mathrm{g}$ for UQ-6, UQ-10, and Vitamin K1, respectively. Both LC-NI-APCI-MS and LC-UV/DAD were applied in the analysis of an activated sludge extract. UQ- $n(n=6-10)$, MK- $n(n=6-10)$, MK- $n\left(\mathrm{H}_{2}\right)(n=7-10)$, MK- $n\left(\mathrm{H}_{4}\right)(n=8-9)$ and MK-8 $\left(\mathrm{H}_{6}\right)$ were detected by LC-NI-APCI-MS, while UQ-6, UQ-7, MK-7 $\left(\mathrm{H}_{2}\right)$, MK-9 and MK-10 $\left(\mathrm{H}_{2}\right)$ were not found by LC-UV/DAD. These results suggest that LC-NI-APCIMS is more sensitive than LC-UV/DAD for the analysis of quinones in environmental samples such as sediment, activated sludge and bio-film in biological processes and other aquatic environments.
\end{abstract}

(c) 2003 Elsevier B.V. All rights reserved.

Keywords: Activated sludge; Environmental analysis; Ubiquinones; Menaquinones

\footnotetext{
*Corresponding author. Tel.: + 86-10-6292-3475; fax: +86-106292-3543.

E-mail address: yangmin@mail.rcees.ac.cn (M. Yang).
}

\section{Introduction}

Microbial ecological studies of activated sludge processes are very important for determining which microbial communities play a significant role in 
wastewater treatment $[1,2]$. In many reports, microbial respiratory quinones have been used as a biomarker for identifying different microbial populations in activated sludge and have been recognised as one of the most important markers in microbial chemotaxonomy [3-5]. Among microbial quinones, there are two major groups of quinones: ubiquinones (related to 1-methyl-2-isoprenyl-3, 4-dimethoxyparabenzoquinone) and menaquinones (related to 1isoprenyl-2-methyl-naphthoquinone) as shown in Fig. $1[3,4]$ and one microbial species usually produces either of them with a certain length of isoprenoid chain [3].

Microbial respiratory quinones have usually been analyzed by liquid chromatography (LC) equipped with a UV, photodiode-array detector after extraction from environmental samples by column chromatography or solid-phase extraction (SPE) [2,4,6-12]. The LC-UV/DAD method is useful for determining the dominating groups in environmental samples. The selectivity of the method, however, is insufficient for environmental samples due to the interference from complex matrices.<smiles>CCCCCC(C)=CCC1=C(C)C(=O)C(OC)=C(C)C1=O</smiles>

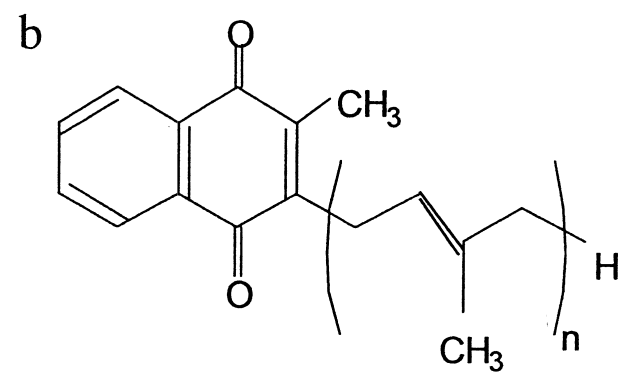

Fig. 1. Quinone structure. (a) Ubiquinone (UQ- $n\left(\mathrm{H}_{x}\right)$ ); (b) menaquinone (MK- $\left.n\left(\mathrm{H}_{x}\right)\right) ; n$ indicates a specified number of isoprene units in the side chain and $x$ denotes the number of hydrogen atoms saturating the isoprenoid chain.
In recent years, LC-MS with various kinds of interface has become an important tool in environmental analyses. If LC-MS could be applied to quinone analysis, the above-mentioned flaws of the LC-UV/DAD method might be overcome because the LC-MS method provides structural information by means of adduct molecular ions and fragment ions. Up until now, only the frit-FAB interface has been explored for the analysis of ubiquinone and menaquinone in pure cultures by LC-MS [13]. The detection sensitivity (detection limit of $40 \mathrm{mg} / \mathrm{l}$ ), however, is not suitable for analyzing microbial communities of complicated environmental samples.

In the present study, we attempted to establish an LC-APCI-MS method with a high sensitivity for analyzing quinone compositions in activated sludge samples.

\section{Experimental}

\subsection{Standard solutions and reagents}

Ubiquinone standard (UQ-6 and UQ-10) and menaquinone standard (Vitamin k1) were obtained from Sigma (St Louis, MO, USA). Standard stock solutions of $1000 \mathrm{mg} / \mathrm{l}$ were prepared in acetone (LC grade, Fisher, China) and they were stored at $4{ }^{\circ} \mathrm{C}$. Working standard solutions were prepared by diluting the stock solutions in acetone and they were stored in the same way.

Methanol, isopropyl ether and hexane were LC grade (Fisher). Ultra pure water was prepared using an Easypure UV Compact Ultrapure System under a conductivity of $18.3 \Omega \mathrm{cm}^{-1}$. The Sep-Pak Plus Silica cartridges $(2 \times 1 \mathrm{~cm}$ I.D.; polypropylene tubes; silica gel amount: $600 \mathrm{mg}$; particle diameter: 55-105 $\mu \mathrm{m})$ were purchased from Waters (Milford, MA, USA).

\subsection{Sample preparation}

Activated sludge samples were taken from the aeration tank in Gaobeidian wastewater treatment plant (Beijing, China). The samples were collected in pre-cleaned plastic bottles, and were pretreated by solid-phase extraction within $1 \mathrm{~h}$.

The procedure reported by $\mathrm{Hu}$ et al. [8] was used 
for sample extraction of ubiquinones and menaquinones with minor modifications. The activated sludge sample ( $0.2 \mathrm{~g}$ dry mass) was centrifuged after $10 \mathrm{ml}$ Ultrapure water and $20 \mathrm{ml}$ chloroform-methanol $(2: 1, \mathrm{v} / \mathrm{v})$ were added to the centrifuge tube. The solution in the centrifuge tube separated into three layers. The top layer (water layer) was discarded, and the middle and bottom layers (activated sludge and chloroform-methanol mixture, respectively) were filtered using glass filter paper. The chloroformmethanol extract was collected, and the filter cake together with the filter paper was then extracted three times with $30 \mathrm{ml}$ chloroform-methanol mixture. The chloroform-methanol extract was pooled and concentrated almost to dryness with the help of a rotary evaporator, and the residues were further extracted
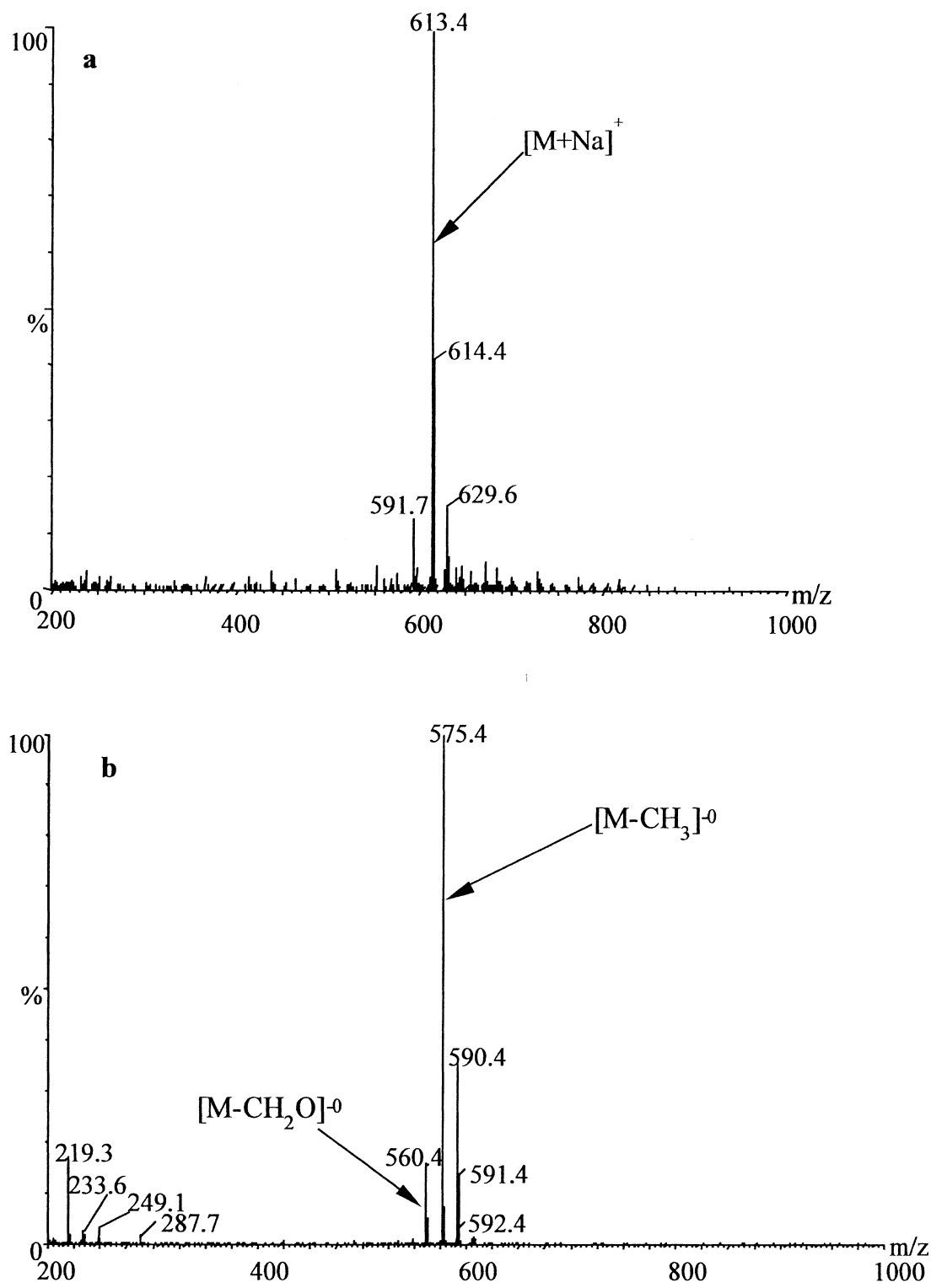

Fig. 2. Mass spectrum of UQ-6: (a) cone voltage of $45 \mathrm{~V}$ in the positive mode; (b) cone voltage of $80 \mathrm{~V}$ in NI mode. UQ-6 concentration: $5 \mathrm{mg} / \mathrm{l}$; injection volume: $20 \mu \mathrm{l}$. 
twice with $20 \mathrm{ml}$ hexane. The hexane extracts were combined.

The hexane extract, containing the ubiquinones and menaquinones, was purified using two Sep-Pak Plus Silica cartridges joined in series. Typically, $5 \mathrm{ml}$ hexane was passed through two Sep-Pak cartridges and a hexane extract was then loaded at a flow of $24 \mathrm{ml} / \mathrm{min}$. Menaquinones were eluted with $20 \mathrm{ml}$ of $2 \%$ diethyl ether in hexane solution from the above Sep-Pak cartridges, and ubiquinones were eluted with $20 \mathrm{ml}$ of $10 \%$ diethyl ether in hexane solution. The eluate of ubiquinones and menaquinones was mixed and dried under a gentle nitrogen stream, and re-dissolved in $2 \mathrm{ml}$ acetone solution for future analysis.

To validate the solid-phase extraction method, standard spiking experiments were carried out by adding standard solutions to $0.2 \mathrm{~g}$ sludge (dry mass) from Kunming lake in Beijing. Before spiking, blank levels were determined and UQ-6, UQ-10 and Vitamin K1 were found to be below detection limits. Triplicate experiments were carried out to determine the precision.

\subsection{Liquid chromatography and mass spectrometry}

Liquid chromatography was carried out on an Alliance 2690 HPLC (Waters, USA) equipped with a quaternary gradient pump, an auto-sampler with 20$\mu l$ injection loop, a photodiode array detector and a reversed-phase column (Zorbax-ODS, $4.6 \mathrm{~mm}$ I.D. $\times$ $250 \mathrm{~mm}$, Alliance and Agilent, USA). A mixture of methanol and isopropyl ether $(83: 17, \mathrm{v} / \mathrm{v})$ was used as the mobile phase at a flow rate of $0.8 \mathrm{ml} / \mathrm{min}$.

A platform ZMD single-quadruple mass spectrometer (Micromass, Manchester, UK) was used with a Z-Spray ion-source fitted with a pneumatically assisted atmospheric pressure chemical ionization probe. The orthogonal Z-Spray interface allowed the entire column effluent from LC to be directed into the source without flow splitting, which enhanced the detection sensitivity. The conditions of mass spectrometry were optimized by direct injection. In the NI mode or positive mode, typical ion source parameters were as follows: APCI capillary voltage at $3.5 \mathrm{kV}$; cone voltage at $45 \mathrm{~V}$; source block temperature at $150{ }^{\circ} \mathrm{C}$; APCI mass spectrometry temperature $350{ }^{\circ} \mathrm{C}$; cone gas flow $300 \mathrm{l} / \mathrm{h}$; desolvation gas flow $400 \mathrm{l} / \mathrm{h}$.

The identification of ubiquinones and menaquinones was carried out in full-scan mode and in total ion-current mode (TIC) by matching the retention time and mass spectrum. The lowest instrument quantitative detection limits $\left(\mathrm{LOD}_{\text {inst }}\right)$ were evaluated by signal-to-noise $(S / N=10)$ in selected ion monitor mode (SIM) and full-scan mode, and the method lowest detection limits $\left(\mathrm{LOD}_{\text {meth }}\right)$ were obtained by correcting $\mathrm{LOD}_{\text {inst }}$ with the recoveries of target compounds and volume of sample. As for the $\mathrm{LOD}_{\text {inst }}$ and the $\mathrm{LOD}_{\text {meth }}$, only UQ-6, UQ-10 and Vitamin K1 were determined because others were not available.

\section{Results and discussion}

\subsection{Analysis of a standard sample mixture}

Fig. 2(a) shows the APCI-MS mass spectrum of UQ-6 in the positive ion mode at a cone voltage of $45 \mathrm{~V}$. The most abundant ion was found at $\mathrm{m} / \mathrm{z}$ 613.4, which corresponds to the sodium adduct ion $[\mathrm{M}+\mathrm{Na}]^{+}$. No in-source fragment ions were observed, even when the cone voltage was increased from 45 to $100 \mathrm{~V}$. This result is quite different from that of FAB, in which $[\mathrm{M}+2]^{2+}$ was found to be the quasi-molecular ion for UQ-6, and the abundant ions were the fragments at $m / z 197$ and 235 [13]. While in the NI mode, the molecular ion $\mathrm{M}^{-0}$ at $\mathrm{m} / \mathrm{z}, 590.4$ was found as the most abundant ion at a cone voltage of $45 \mathrm{~V}$, which was different from the conventional quasi-molecular ion $[\mathrm{M}-\mathrm{H}]^{-}$. This behavior was also reported in LC-NI-APCI-MS of nitro-polycyclic aromatic hydrocarbons [14]. In addition, when the cone voltage was gradually increased from 45 to 100 $\mathrm{V}$, fragment ions by in-source collision induced dissociation(CID) were found. Fig. 2(b) shows the mass spectrum of UQ- 6 at a cone voltage of $80 \mathrm{~V}$. In addition to the molecular ion $\mathrm{M}^{-0}$ at $\mathrm{m} / z$. 590.4 with $38 \%$ relative abundance, we also found the base peak ion at $\mathrm{m} / \mathrm{z} 575.4\left(\left[\mathrm{M}-\mathrm{CH}_{3}\right]^{-0}\right)$ together with two other fragment ions at $\mathrm{m} / \mathrm{z} 560.4\left(\left[\mathrm{M}-\mathrm{CH}_{2} \mathrm{O}\right]^{-0}\right)$ and 219.3 with a relative abundance of 18 and $19 \%$, respectively.

The mass spectra of Vitamin K1 were also investi- 
gated using positive ion mode and NI-APCI mode. No response was found when Vitamin K1 was injected at a concentration of $10 \mathrm{mg} / 1$ in positive ion mode. However, when the cone voltage was increased to $80 \mathrm{~V}$, besides the molecular ion at $\mathrm{m} / \mathrm{z}$ 450.7, the NI-APCI mass spectrum had two fragment ions at $\mathrm{m} / \mathrm{z} 210.2$ and 225.5 with a relative abundance of 38 and $45 \%$. Thus, LC-NI-APCI-MS provides the most useful information for the analysis of UQ-6 and Vitamin K1.

Finally, the possibility of using the ESI-MS method for quinone analysis was also investigated in this study. However, the sensitivity in both positive and NI modes was very low.

\subsection{Method validation}

The average recoveries of UQ-6, UQ-10 and Vitamin K1 in environmental samples are $93 \pm 12$, $95 \pm 6$ and $98 \pm 10 \%$. Moreover, this indicates that the LC-NI-APCI-MS method performed satisfactorily despite interference by environmental matrices. Relative standard deviations (RSDs) of the triplicate analysis of spiked samples ranged from 3 to $12 \%$. The between-day reproducibility of environmental sample was determined with triplicate detection over
5 days and the RSDs for UQ-6, UQ-10 and Vitamin $\mathrm{K} 1$ were below $12 \%$.

The $\mathrm{LOD}_{\text {inst }}$ in the positive mode were estimated to be 4 and $50 \mathrm{ng}$ for UQ-6 and UQ-10, respectively, when the injection volume is $20 \mu$ l. Although the sensitivity for analyzing quinones even in the positive mode was significantly improved, compared to frit-FAB-LC-MS (typically $40 \mathrm{mg} / 1$ ) [13], the dayto-day response varied markedly. The $\mathrm{LOD}_{\text {inst }}$ of UQ-6, UQ-10 and Vitamin K1 were also established in the NI mode SIM. The LOD inst $_{\text {for UQ-6, UQ-10 }}$ and Vitamin K1 in the NI mode were 0.4, 4 and 0.12 ng $(S / N=10)$, respectively. Considering the weight of sludge sample ( $0.2 \mathrm{~g}$ dry mass) and the recoveries of target compounds, the $\mathrm{LOD}_{\text {meth }}$ achieved by the spiking experiment were estimated to be 0.2, 2 and $0.06 \mu \mathrm{g} / \mathrm{g}$ for UQ-6, UQ-10 and Vitamin K1, respectively. On the other hand, the $\mathrm{LOD}_{\text {inst }}$ for UQ-6, UQ-10 and Vitamin K1 were estimated to be 10,100 and $2 \mathrm{ng}(S / N=10)$ using LC-NI-APCI-MS in full-scan mode, and $\mathrm{LOD}_{\text {meth }}$ were estimated to be 7, 60 and $1.2 \mu \mathrm{g} / \mathrm{g}$ for UQ-6, UQ-10 and Vitamin $\mathrm{K} 1$, respectively. Unfortunately, no more quinones except UQ-6, UQ-10 and Vitamin K1, could commercially be purchased, and we are trying to fractionate these quinones from biological samples.

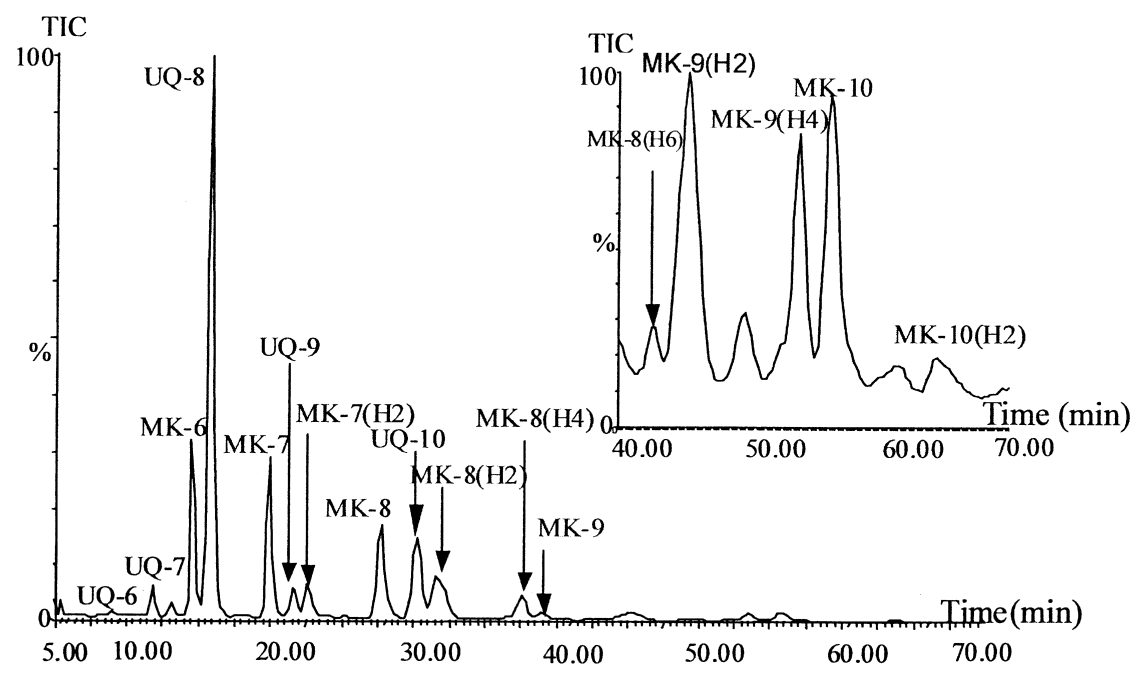

Fig. 3. LC-NI-APCI-MS chromatogram of the sample extracted from activated sludge in an aeration tank. APCI capillary voltage: $3.5 \mathrm{kV}$; cone voltage: $45 \mathrm{~V}$; source block temperature: $150{ }^{\circ} \mathrm{C}$; APCI mass spectrometry temperature: $350{ }^{\circ} \mathrm{C}$; cone gas flow: $3001 / \mathrm{h}$; desolvation gas flow: $400 \mathrm{l} / \mathrm{h}$; injection volume: $20 \mu \mathrm{l}$. 


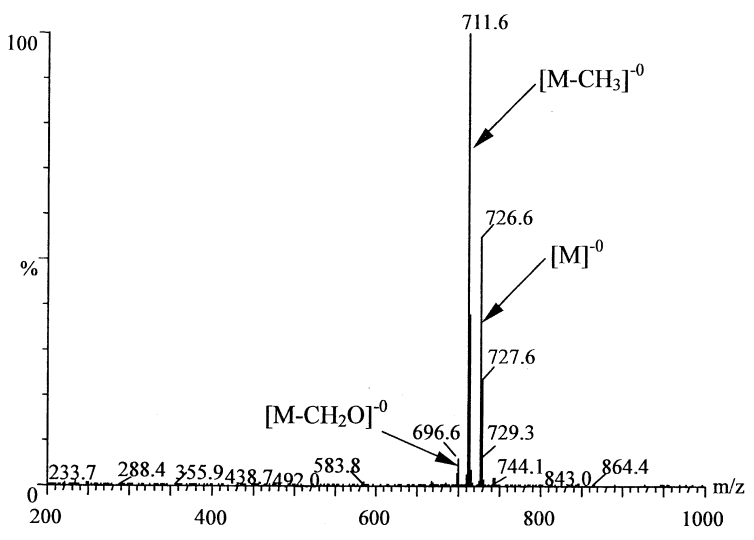

Fig. 4. Mass spectrum of UQ-8 in the sample corresponding to Fig. 3. Cone voltage: $80 \mathrm{~V}$; other analytical conditions, see Fig. 3.

\subsection{Analyses of the microbiological community}

The LC-NI-APCI-MS method established above was applied to analyze the activated sludge sample. Seventeen kinds of quinones were observed in the NI mode (Fig. 3) and five peaks were identified as UQ- $n$ $(n=6-10)$ by the molecular ions $\mathrm{M}^{-0}$ at $\mathrm{m} / \mathrm{z} 590.4$, 658.5, 726.6, 794.6 and 862.7, and corresponding fragment ions. Fig. 4 shows the mass spectrum of UQ-8. The molecular ion of $\mathrm{M}^{-0}$ at $\mathrm{m} / \mathrm{z} 726.6$, the base peak ion at $m / z 711.6\left(\left[\mathrm{M}-\mathrm{CH}_{3}\right]^{-0}\right)$ and the fragment ion at $m / z 696.6\left(\left[\mathrm{M}-\mathrm{CH}_{2} \mathrm{O}\right]^{-0}\right)$ proved that this peak was UQ-8. Similarly, the other 12 peaks were identified as MK- $n(n=6-10)$, MK- $n$ $\left(\mathrm{H}_{2}\right)(n=7-10)$, MK- $n\left(\mathrm{H}_{4}\right)(n=8-9)$ and MK-8 $\left(\mathrm{H}_{6}\right)$ by the molecular ions $\mathrm{M}^{-0}$ and fragment ions. Thus, the results show that the LC-NI-APCI-MS method provided useful structural information for identifying the ubiquinones and menaquinones in environmental samples. However, the above identifications for the new compounds need to be further confirmed with their standards or other methods such as NMR.

The wavelengths used to measure quinones were 275 and $270 \mathrm{~nm}$ for ubiquinones and menaquinones, respectively [2,5,8-12]. Fig. 5 shows the LC-UV/ DAD chromatogram of the above-mentioned sample at $270 \mathrm{~nm}$. Menaquinones, such as MK- $n(n=6-8$, 10), MK-8 $\left(\mathrm{H}_{2}, \mathrm{H}_{4}, \mathrm{H}_{6}\right)$, and MK-9 $\left(\mathrm{H}_{2}, \mathrm{H}_{4}\right)$, were found in the LC-UV/DAD chromatogram based on the individual characteristic UV spectrum [13]. However, no peaks for MK-9, MK-7 $\left(\mathrm{H}_{2}\right)$ and MK-10 $\left(\mathrm{H}_{2}\right)$ were found. In addition, compounds like MK-8 $\left(\mathrm{H}_{2}\right)$ and MK-8 $\left(\mathrm{H}_{4}\right)$ cannot be quantified with the LC-UV/DAD method, due to the interference from sample matrices, even when they would be available as standards. Similarly, only UQ- $n(n=8-10)$ was found in the LC-UV/DAD chromatogram at 275 $\mathrm{nm}$. Hence, LC-NI-APCI-MS is a more sensitive and specific method than LC-UV/DAD for analyzing microbial quinones in activated sludge.

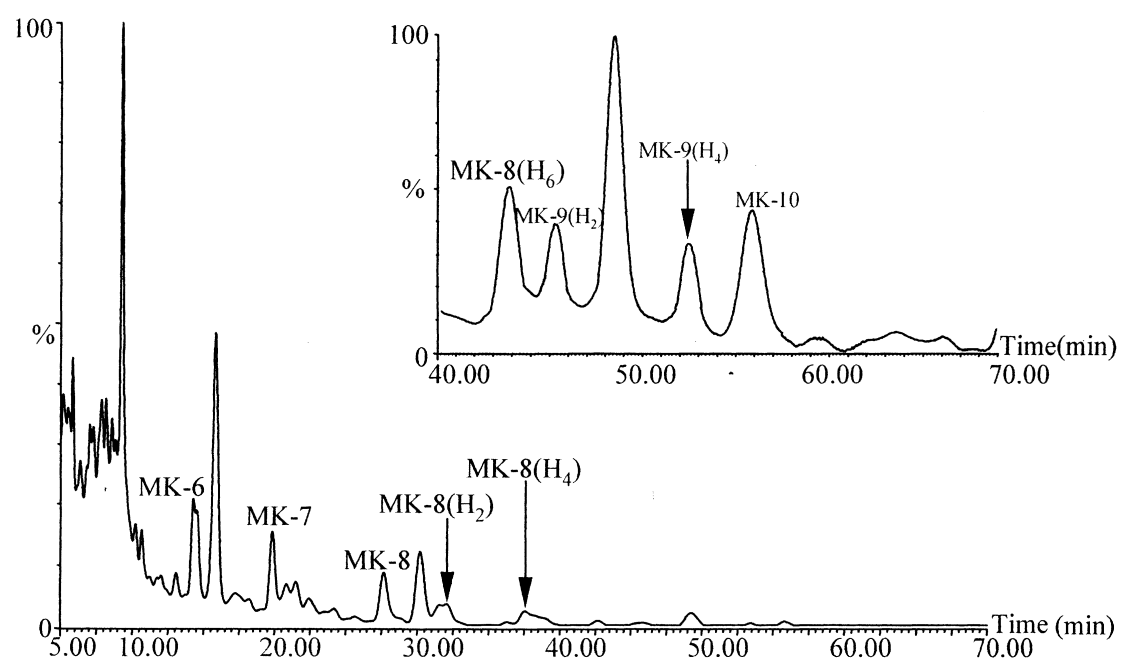

Fig. 5. LC-UV/DAD chromatogram of the sample corresponding to Fig. 3 at $270 \mathrm{~nm}$. 


\section{Conclusions}

An LC-NI-APCI-MS method with satisfactory sensitivity and selectivity was established for qualitatively analysing ubiquinones and menaquinones in environmental samples. This technique overcomes the shortcomings of LC-UV/DAD and frit-FABLC-MS methods. The LC-NI-APCI-MS method for identification of ubiquinones and menaquinones seems to be an effective tool for the analysis of the microbial community in environmental samples.

\section{Acknowledgements}

Funding for this research was provided by the Chinese Academy of Sciences (Hundred-Talents Program) and the Key Laboratory of Environmental Science and Technology of High Education of Henan Province. Special thanks are extended to Professor $\mathrm{Hu}$ Hongying of Tsinghua University for his valuable advice in an initial study.

\section{References}

[1] W.O. Pipes, Adv. Appl. Microbiol. 8 (1966) 77.
[2] A. Hiraishi, K. Masamune, H. Kitamura, Appl. Environ. Microbiol. 55 (1989) 897.

[3] M.D. Collins, D. Jones, Microbiol. Rev. 45 (1981) 316.

[4] D.B. Hedrick, D.C. White, J. Microbiol. Methods 5 (1986) 243.

[5] A. Hiraishi, J. Gen. Appl. Microbiol. 34 (1988) 39.

[6] M.D. Collins, D. Jones, J. Appl. Bacteriol. 51 (1981) 129.

[7] J. Tamaoka, Y. Katayama-Fujimura, H. Kuraishi, J. Appl. Bacteriol. 54 (1983) 31.

[8] H.Y. Hu, K. Fujie, K. Urano, J. Biosci. Bioeng. 87 (1999) 378.

[9] H.Y. Hu, B.R. Lim, N. Goto, V.K. Bhupathiraju, K. Fujie, Water Sci. Technol. 43 (2001) 99.

[10] H.Y. Hu, K. Fujie, H. Nakagome, K. Urano, A. Katayam, Water Res. 33 (1999) 3263.

[11] K. Fujie, H.Y. Hu, H. Tanaka, K. Urano, K. Saito, A Katayama, Soil Sci. Plant Nutr. 44 (1998) 393.

[12] H.Y. Hu, K. Fujie, K. Urano, J. Ferment. Bioeng. 78 (1994) 100.

[13] M. Nishijima, M. Araki-Sakai, H. Sano, J. Microbiol. Methods 28 (1997) 113.

[14] H. Moriwaki, K. Funasawa, M. Uebori, Anal. Sci. 16 (2000) 1247. 